\title{
Surface quality and surface waves on subwavelength-structured silver films
}

\author{
G. Gay, O. Alloschery, and J. Weiner* \\ IRSAMC/LCAR, Université Paul Sabatier, 118 route de Narbonne, 31062 Toulouse, France
}

H. J. Lezec

Thomas J. Watson Laboratories of Applied Physics, California Institute of Technology, Pasadena, California 91125, USA and Centre National de la Recherche Scientifique, 3, rue Michel-Ange, 75794 Paris cedex 16, France

C. O’Dwyer

Tyndall National Institute, University College Cork, Cork, Ireland

M. Sukharev and T. Seideman

Department of Chemistry, Northwestern University, 2145 Sheridan Road, Evanston, Illinois 60208-3113, USA

(Received 25 August 2006; published 24 January 2007)

\begin{abstract}
We analyze the physical-chemical surface properties of single-slit, single-groove subwavelength-structured silver films with high-resolution transmission electron microscopy and calculate exact solutions to Maxwell's equations corresponding to recent far-field interferometry experiments using these structures. Contrary to a recent suggestion the surface analysis shows that the silver films are free of detectable contaminants. The finite-difference time-domain calculations, in excellent agreement with experiment, show a rapid fringe amplitude decrease in the near zone (slit-groove distance out to 3-4 wavelengths). Extrapolation to slit-groove distances beyond the near zone shows that the surface wave evolves to the expected bound surface plasmon polariton (SPP). Fourier analysis of these results indicates the presence of a distribution of transient, evanescent modes around the SPP that dephase and dissipate as the surface wave evolves from the near to the far zone.
\end{abstract}

DOI: 10.1103/PhysRevE.75.016612

The optical response of subwavelength-structured metallic films has enjoyed a resurgence of interest in the past few years due to the quest for an all-optical solution to the inexorable drive for ever-smaller, more densely integrated devices operating at ever-higher bandwidth. Two basic questions motivate research in this field: how to confine micrometer light waves to subwavelength dimensions [2] and how to transmit this light without unacceptable loss over at least tens of micrometers $[3,4]$. Although surface waves and in particular periodic arrays of surface plasmon polaritons (SPPs) have received a great deal of attention as promising vehicles for subwavelength light confinement and transport, detailed understanding of their generation and early time evolution (within the first few wave periods) in and on real metal films is still not complete [5-8].

Recent measurements [1,9] of far-field interference fringes arising from surface wave generation in single slitgroove structures on silver films have characterized the amplitude, wavelength, and phase of the surface waves. After a rapid amplitude decrease within the first $3 \mu \mathrm{m}$ from the generating groove, waves persisting with near-constant amplitude over tens of micrometers were observed. Such longrange transport is the signature of a "guided mode" SPP, but the measured wavelength was found to be markedly shorter than the expectation from conventional theory [10]. One possible reason advanced for the disparity between experiment and theory was the presence of an oxide or sulfide dielectric layer on the silver surface, and it has been suggested [11] that an $11 \mathrm{~nm}$ layer of silver sulfide would bring experiment

\footnotetext{
*Electronic address: jweiner@irsamc.ups-tlse.fr
}

PACS number(s): 42.25.Fx, 73.20.Mf, 78.67.-n

and theory into agreement. We report here the results of two investigations: one experimental, into the physical-chemical surface properties of the silver structures, and the other theoretical, into the calculated optical response of the silver slitgroove structures using the finite-difference time-domain (FDTD) technique to numerically solve Maxwell's equations. These studies show no evidence of oxide or sulfide layers on the silver surface and the numerical solutions to Maxwell's equations not only show good agreement with measurements reported in [1] but also point to the important role played by short-range evanescent components in the early time evolution of the surface wave.

Three typical structured samples were chosen for examination with fabrication dates of about 12 months, 6 months, and 1 week from the date of the transmission electron microscopy (TEM) analysis. The structures dating from 6 months and 12 months were part of the series actually used in the previous reports [1,9]. They consist of a $400 \mathrm{~nm}$ silver film evaporated onto a fused silica substrate (Corning 7980 uv grade $25 \mathrm{~mm}^{2}, 1 \mathrm{~mm}$ thick, optically polished on both sides to a roughness of no more than $0.7 \mathrm{~nm}$ ). The structured substrates are stored in Fluoroware sample holders, $25 \mathrm{~mm}$ diameter and $1 \mathrm{~mm}$ in depth at the center. Electrontransparent sections for cross-sectional transmission electron microscopy examination were prepared by sample thinning to electron transparency using standard focused $\mathrm{Ga}^{+}$-ion beam (FIB) milling procedures [12] in a FEI 200 FIB workstation and placed on a holey carbon support. The TEM characterization was performed using a Philips CM300 Schottky field emission gun (FEG) microscope operating at $300 \mathrm{kV}$. The FEGTEM has a point resolution of $0.2 \mathrm{~nm}$ and an information limit of $0.12 \mathrm{~nm}$. The minimum focused electron beam probe size is $0.3 \mathrm{~nm}$. In dark-field images diffracted 


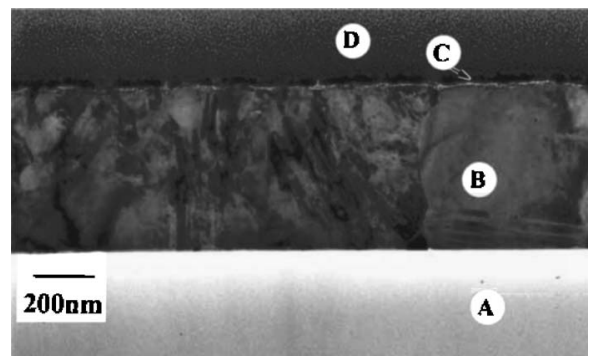

FIG. 1. Cross-sectional bright-field through-focal TEM micrograph of the Ag layer on a fused silica substrate. Labels refer to fused silica substrate A, silver layer B, capping gold layer C, and capping platinum layer D. The darkened features in the Ag layer (B) are dislocations and grain boundaries.

flux passes the objective aperture while the direct straightthrough beam is blocked. The diffracted beam interacts strongly with the specimen and selection of a particular diffracted beam allows better visual phase differentiation. Typical regions of $\mathrm{Ag}$ layer that were thinned to electron transparency are shown at relatively low magnification in the bright-field micrograph in Fig. 1. An immediate distinction can be made between the fused silica substrate (A) and the $\mathrm{Ag}$ deposit (B). Protective capping layers of $\mathrm{Au}$ and $\mathrm{Pt}$, applied at the time of analysis, are marked at $\mathrm{C}$ and $\mathrm{D}$, respectively. The latter are used to prevent any "top down" ion damage of the cross section during the ion beam thinning preparation. The white line marked by an arrow in Fig. 1 is a band that contains very fine particles of Au formed during the initial stages of the deposition of this metal. The fine particles do not exhibit the same degree of absorption contrast as the bulk of the Au above them because the particle diameters are less than the thickness of the cross-sectional slice and thus do not extend through the full thickness of the TEM sample. It is clearly observed that the Au nanoparticles form a separate layer above the Ag deposit. Local undulations in the Ag layer are observed to be devoid of any oxide or sulfide layer. Fresnel contrast methods were also used to examine the upper surface regions of the silver at the detail shown in Fig. 2. Imaging of layers in cross section always results in Fresnel fringes. Because the amplitude and phase changes that occur when an electron is scattered elastically are characteristic of the atomic number, there will be changes in the elastic scattering directly related to the form of the projected scattering potential when there is a composition

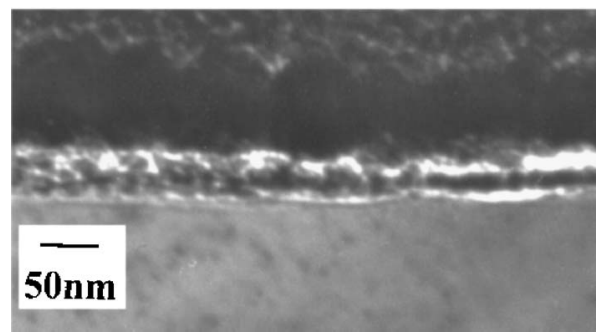

FIG. 2. A higher-magnification defocused image of the upper surface showing the Au-particle-containing protection layer. No Fresnel fringes are observed at the uppermost Ag surface when compared with in-focus images. White spaces indicate voids. change at an interface viewed in projection [13], analogous to a phase grating filtering of the scattered electronic wave function. Interface Fresnel effects can thus provide a signature of the form and magnitude of any compositional discontinuity that is present. The visibility of these fringes depends on the thickness of the specimen and on the defocus value of the microscope. From Fig. 2, the critical observation is the absence of Fresnel effects at the surface of the metal other than at the localized regions associated with the gold protective coating. The lack of Fresnel fringes at the upper surface indicates a lack of variation in the scattering potentials and hence of the chemical composition at the interface. The silver surface is not covered by a detectable layer of sulfide or oxide since their presence would exhibit differences in scattering potential by comparison with the metal itself [14]. The lower detection limit for such compositional differences is a few tenths of a nanometer layer thickness. The absence of a dielectric layer is not surprising when account is taken of the small-volume, air-tight sample storage and the trace fractional concentrations of sulfur-containing contaminants in ordinary laboratory air [15].

The optical response of structured metal surfaces is simulated using a finite-difference time-domain approach [16]. The subwavelength slit-groove structures $[1,9]$ are modeled in two dimensions and excited by TM polarized light. In metallic regions of space $\varepsilon$ is a complex, frequencydependent function. Within the standard Drude model [17] it is given as

$$
\varepsilon(\omega)=\varepsilon_{0}\left(\varepsilon_{\infty}-\frac{\omega_{p}^{2}}{\omega^{2}+i \Gamma \omega}\right),
$$

where $\varepsilon_{0}$ is the electric permittivity of free space, $\varepsilon_{\infty}=\varepsilon(\omega$ $\rightarrow \infty$ ) the dimensionless infinite-frequency limit of the dielectric constant, $\omega_{p}$ the bulk plasmon frequency, and $\Gamma$ the damping rate. We numerically fit the real and imaginary parts of the Drude dielectric constant in the form of Eq. (1) to the experimental data collected in [18]. The Drude parameters obtained in the wavelength regime ranging from 750 to $900 \mathrm{~nm}$ for silver are $\varepsilon_{\infty}=3.2938, \omega_{p}=1.3552 \times 10^{16} \mathrm{rad} \mathrm{s}^{-1}$, and $\Gamma=1.9944 \times 10^{14} \mathrm{rad} \mathrm{s}^{-1}$, which correspond to $\operatorname{Re}[\varepsilon]$ $=-33.9767$ and $\operatorname{Im}[\varepsilon]=3.3621$ at $\lambda_{0}=852 \mathrm{~nm}$. These fitted parameters are close to those determined in [1] by ellipsometry, $\operatorname{Re}[\varepsilon]=-33.27$ and $\operatorname{Im}[\varepsilon]=1.31$. Numerical results were not sensitive to this range of parameter variability. For reference we also implement perfect electric conductor (PEC) boundary conditions, where the metal dielectric constant is set to negative infinity, and all electromagnetic field components are therefore strictly zero in metal regions. The light source is a plane wave $E_{\text {inc }}$, incident perpendicular to the metal surface and depending on time as $E_{\text {inc }}(t)$ $=E_{0} f(t) \cos \omega t$, where $E_{0}$ is the peak amplitude of the pulse, $f(t)=\sin ^{2}(\pi t / \tau)$ is the pulse envelope, and $\tau$ is the pulse duration $[f(t>\tau)=0]$. Time propagation is performed by a leapfrogging technique [16]. In order to prevent nonphysical reflection of outgoing waves from the grid boundaries, we employ perfectly matched layer (PML) absorbing boundaries. To avoid accumulation of spurious electric charges at the ends of the excitation line and generate a pure plane 


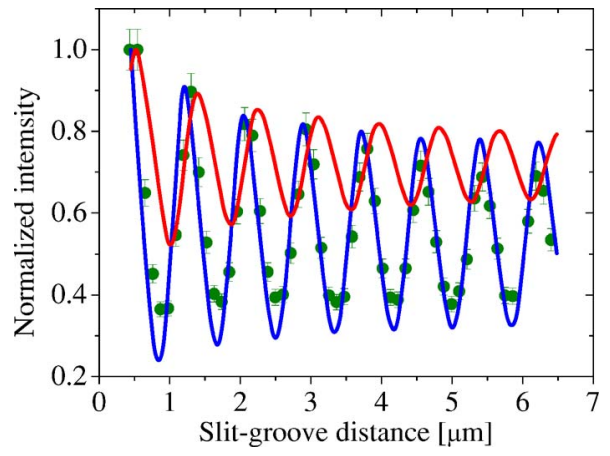

FIG. 3. (Color online) Comparison of FDTD simulation and experiment. Green points are experimental data taken from [1]. Blue solid curve that closely fits the points corresponds to Drude model; red solid curve with longer fringe wavelength and smaller amplitude than data corresponds to PEC model.

wave with a well-defined incident wavelength, we embed the ends in the PML regions. Convergence is achieved with a spatial step size of $\delta x=\delta y \leqq 4 \mathrm{~nm}$ and a temporal step size of $\delta t=\delta x /(2 c)$, where $c$ denotes the speed of light in vacuum. The parallel computation technique used in our simulations is described in detail in [21]. Calculations of the intensity $I$ $\sim\left(E_{x}^{2}+E_{y}^{2}+H_{z}^{2}\right)$ are performed and converged along a box contour.

The collected data are averaged over time and the spatial coordinates for a range of slit-groove distances. Finally, the space- and time-averaged intensity is normalized to unit maximum. Our results are converged to the $\mathrm{cw}$ limit with incident pulse durations $\tau \gtrsim 200 \mathrm{fs}$. A direct comparison of the experimental data with both the PEC and the Drude models is shown in Fig. 3. Clearly, the Drude model agrees very well with the data, whereas the PEC model predicts oscillations of the intensity with a noticeably larger wavelength and smaller amplitude. In order to properly determine the wavelength of oscillations we use a cosine function with an exponentially decreasing amplitude plus a constant offset to fit the data shown in Fig. 3. We also extend both the FDTD simulation and the fitting function to a slit-groove distance of $16 \mu \mathrm{m}$, well beyond the range of experimental data, and take the Fourier transform of the fitted function over this extended range. We obtain the associated power spectrum expressed as a wavelength distribution. The results are shown in Fig. 5 below:

$$
I_{\text {fit }}(x)=\left[A_{1}+A_{2} \exp \left(A_{3} x\right)\right] \cos \left(A_{4} x+A_{5}\right)+A_{6} .
$$

The FDTD simulation and analytic fit for larger slitgroove distances are shown in Fig. 4 for the case of the Drude model. The fitting function Eq. (2) tracks the FDTD results over the entire slit-groove distance range. Figure 5 shows the normalized power spectrum corresponding to Eq. (2) as a function of the wavelength. The two power spectrum plots of Fig. 5 provide the effective wavelength $\lambda_{\text {eff }}$ at which surface waves propagate as well as the distribution of modes around the peak. Note that the Drude model for silver shows a marked blueshift in peak wavelength and a noticeable broadening of the distribution compared to the perfect metal PEC model. The Drude simulation results in $\lambda_{\text {eff }}$

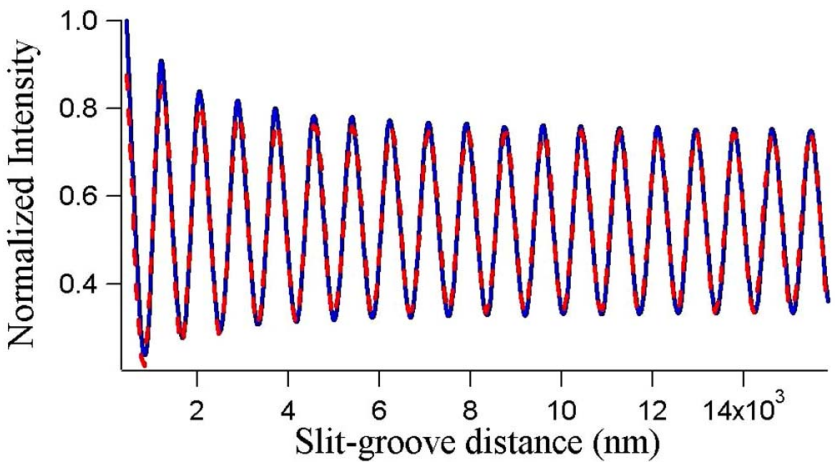

FIG. 4. (Color online) Blue solid curve plots the same Drude model FDTD calculation as in Fig. 3 but extended to $16 \mu \mathrm{m}$ slitgroove distance. Red dashed curve plots the fitting function of Eq. (2).

$=837.482 \mathrm{~nm}$, whereas the PEC gives $\lambda_{\text {eff }}=852.066 \mathrm{~nm}$, very close to the free-space reference wavelength $\lambda_{0}$ $=852 \mathrm{~nm}$. The effective surface index of refraction, $n_{\text {eff }}$ $=\lambda_{0} / \lambda_{\text {eff }}$, leads to the following values: $n_{\text {eff }}($ Drude $)=1.0173$ and $n_{\text {eff }}(\mathrm{PEC})=0.9999$.

In summary, surface analysis by transmission electron microscopy of subwavelength-structured silver films used to investigate their optical response [1,9] showed no detectable evidence of material on the surface other than silver. The suggestion that an $11 \mathrm{~nm}$ sulfide layer may be present so as to bring the interference pattern calculated by the authors of Ref. [11] into agreement with experiment is therefore not confirmed. In contrast to the calculations reported by Ref. [11], the numerical solution of Maxwell's equations reported here shows excellent agreement with the fringe amplitude and wavelength over the near-zone slit-groove range measured in [1]. We emphasize that the amplitude decrease in this near-zone reflects evanescent mode dephasing and dissipation. It is much faster than loss rates expected from surface scattering or absorption [1]. Extrapolation of the FDTD simulations beyond the range of the measurements shows that the initially decreasing amplitude of the fringe settles to an oscillation with near-constant amplitude and fringe contrast. These features resemble the calculations of [11], but the

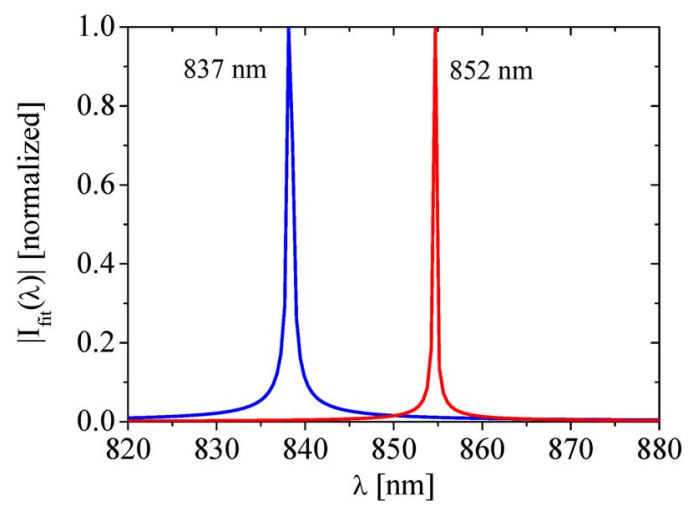

FIG. 5. (Color online) Fourier transform spectra of Eq. (2) for Drude peaked at $837 \mathrm{~nm}$ (blue) and PEC peaked at $852 \mathrm{~nm}$ (red) FDTD simulation fits. Indicated $\lambda_{\text {peak }}$ correspond to $n_{\text {eff }}$ (Drude) $=1.017, n_{\mathrm{eff}}(\mathrm{PEC})=1.000$. 
results reported here accord with experiment without the need to invoke an $11 \mathrm{~nm}$ silver sulfide layer. Fourier analysis of the FDTD simulations reveal that the most probable wavelength in the Fourier distribution is $837 \mathrm{~nm}$, within about $2 \mathrm{~nm}$ of the expected long-range SPP wavelength of $839 \mathrm{~nm}$. Similar analysis of the PEC simulations yields a peak wavelength, as expected, at the free-space wavelength of $852 \mathrm{~nm}$. Finally, Figs. 4 and 5 point to the important contributions to the surface wave of transient modes neighboring the SPP at slit-groove distances within the near zone. The presence of these modes implies a surface $k$-mode "wave packet" that evolves to the final SPP as the wave passes beyond the near zone. This near zone, however, extends over several wavelengths; and therefore any theory of subwavelength array transmission must take these ephemeral, evanescent modes into account.
Support from the Ministère délégué à l'Enseignement supérieur et à la Recherche under the programme ACI "Nanosciences-Nanotechnologies," the Région MidiPyrénées (Grant No. SFC/CR 02/22), and FASTNet (Grant No. HPRN-CT-2002-00304) EU Research Training Network, is gratefully acknowledged, as is support from the Caltech Kavli Nanoscience Institute, the AFOSR under Plasmon MURI Grant No. FA9550-04-1-0434, the National Energy Research Scientific Computing Center, the U.S. Department of Energy under Contract No. DE-AC03-76SF00098, and the San Diego Supercomputer Center under Grant No. PHY050001. Discussions with P. Lalanne, M. Mansuripur, and $\mathrm{H}$. Atwater and computational assistance from Y. Xie are also gratefully acknowledged.
[1] G. Gay et al., Nat. Phys. 2, 262 (2006).

[2] J. A. Dionne, L. A. Sweatlock, H. A. Atwater, and A. Polman, Phys. Rev. B 73, 035407 (2006), and references therein.

[3] R. Zia, M. D. Selker, and M. L. Brongersma, Phys. Rev. B 71, 165431 (2005).

[4] W. L. Barnes, A. Dereux, and T. W. Ebbesen, Nature (London) 424, 824 (2003), and references therein.

[5] Y. Xie et al., Opt. Express 13, 4485 (2005).

[6] A. R. Zakharian, J. V. Moloney, and M. Mansuripur, Opt. Express 15, 183 (2007).

[7] Q. Cao and P. Lalanne, Phys. Rev. Lett. 88, 057403 (2002).

[8] P. Lalanne, J. P. Hugonin, and J. C. Rodier, Phys. Rev. Lett. 95, 263902 (2005).

[9] G. Gay, O. Alloschery, B. Viaris de Lesegno, J. Weiner, and H. J. Lezec, Phys. Rev. Lett. 96, 213901 (2006).

[10] H. Raether, Surface Plasmons on Smooth and Rough Surfaces and on Gratings (Springer-Verlag, Berlin, 1988).
[11] P. Lalanne and J. P. Hugonin, Nat. Phys. 2, 556 (2006).

[12] L. A. Giannuzzi and F. A. Stevie, Micron 30, 197 (1999).

[13] Z. L. Wang, Elastic and Inelastic Scattering in Electron Diffraction and Imaging (Plenum, New York, 1995).

[14] B. Fultz and J. Howe, Transmission Electron Microscopy and Diffractometry of Materials, 2nd ed. (Springer, New York, 2005).

[15] See G. Gay et al., e-print physics/0608116 for details.

[16] A. Taflove and S. C. Hagness, Computational Electrodynamics: The Finite-Difference Time-Domain Method (Artech House, Boston, 2000).

[17] C. F. Bohren and D. R. Huffman, Absorption and Scattering of Light by Small Particles (Wiley, New York, 1983).

[18] D. W. Lynch and W. R. Hunter, Handbook of Optical Constants of Solids (Academic, Orlando, FL, 1985).

[21] M. Sukharev and T. Seideman, J. Phys. Chem. 124, 144707 (2006). 